\title{
Minimal model of a heat engine: An information theory approach
}

\author{
Yun Zhou and Dvira Segal \\ Chemical Physics Theory Group, Department of Chemistry, \\ University of Toronto, 80 Saint George St. Toronto, Ontario, Canada M5S $3 H 6$
}

\begin{abstract}
We construct a generic model for a heat engine using information theory concepts, attributing irreversible energy dissipation to the information transmission channels. Using several forms for the channel capacity, classical and quantum, we demonstrate that our model recovers both the Carnot principle in the reversible limit, and the universal maximum power efficiency expression of nonreversible thermodynamics in the linear response regime. We expect the model to be immensely useful as a testbed for studying fundamental topics in thermodynamics.
\end{abstract}

PACS numbers: 05.70.-a, 05.70.Ln, 89.70.-a, 89.70.Kn

Introduction. - The Maxwell's demon puzzle, suggesting a violation of the second law of thermodynamics, has been exorcised using Landauer's memory erasure principle [1]. Treating the demon's intelligence as information has manifested a fundamental connection between information and physics, revealing the specific role of memory in terms of the second law of thermodynamics [2].

The goal of this paper is to suggest a minimal-universal model of a heat engine (demon) using information theory concepts, and to employ it for analyzing the operation principles of finite-time thermodynamic machines [3]. In our engine irreversible energy dissipation is attributed to information transfer within the demon's channels, compensating entropy decrease in the system. The model serves as a testbed for studying fundamental topics in thermodynamics: The universality of the maximum power efficiency and its relationship to the Carnot efficiency.

A central topic in thermodynamics is the study of the efficiency of thermal engines, manifesting universal features. According to the Carnot result the efficiency (work output over heat input) of a cyclic thermal machine, operating between two heat baths temperatures $T_{C}$ and $T_{H}$ $\left(T_{C}<T_{H}\right)$, is at most

$$
\eta_{C}=1-\frac{T_{C}}{T_{H}} .
$$

The upper limit is obtained for engines that work reversibly. However, as reversible processes occur infinitesimally slowly, the power produced is zero. Operating away from equilibrium, a more practical question is the efficiency at maximum power, $\eta_{M}$, optimizing an engine cycle with respect to its power rather than efficiency. For a specific model, Curzon and Ahlborn (CA) derived the maximum power efficiency [4]

$$
\begin{aligned}
\eta_{C A} & =1-\sqrt{\frac{T_{C}}{T_{H}}}=1-\sqrt{1-\eta_{C}} \\
& \approx \eta_{C} / 2+\eta_{C}^{2} / 8+\mathcal{O}\left(\eta_{C}^{3}\right),
\end{aligned}
$$

relaying on the endoreversible approximation in which the sole source of irreversibility is due to heat transfer processes [3]. Does (2) represent a universal characteristic of finite-time thermodynamics, or does it depend

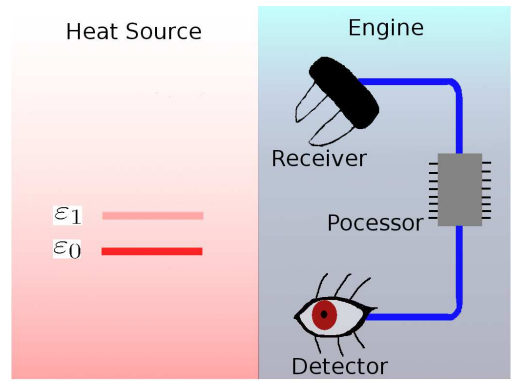

FIG. 1: A schematic representation of the model heat engine, see description in the text.

on the specific model? In the linear response regime it has been recently proven that the efficiency at maximum power is upper bounded by (2), which in this regime is exactly half of the Carnot efficiency [5]. The upper limit is reached for a specific class of "strongly coupled" models for which the energy flux is directly proportional to the work-generating flux. In contrast, in the nonlinear regime general results are missing, and expressions deviating from the CA efficiency were reported [6, 7].

In the present model irreversible loss of energy occurs at the communication channels, transmitting classical information (entropy) from a heat source $\left(T_{H}\right)$ to the engine $\left(T_{C}\right)$. We consider both classical and quantum channels, encoding classical information in quantum states, and show that in both cases Carnot efficiency can be achieved when the device works reversibly. We also demonstrate that in the linear regime (small temperature differences) the efficiency at maximum power is $\eta_{C} / 2$. Using a generic form for the channel capacity we show that an agreement to higher terms in (2) can be obtained for a class of channels. Our study indicates on the fundamental status of the (linear) CA efficiency in irreversible thermodynamics. We find that if a model obeys the Carnot limit for a reversible process, it must also follow, for a nonreversible operation, the universal behavior, $\eta_{M}=\eta_{C} / 2$ in the linear response regime, and vice versa. Moreover, we find that the ratio of $1 / 2$ between the maximum efficiency and the max-power efficiency holds even below the theoretical upper bounds, for imperfect systems. 
Model. - Our model includes a finite subsystem immersed in a heat bath and an engine (demon), see Fig. 1. In particular, the subsystem may include two discrete states 0 and 1 of energies $\varepsilon_{0}=0$ and $\varepsilon_{1}=\varepsilon$ respectively. The subsystem is assumed to be tightly attached to a heat bath at temperature $T_{H}$, thus in thermal equilibrium the levels population follows the Boltzmann distribution $P_{0}=\frac{1}{1+\exp \left(-\varepsilon / T_{H}\right)}, P_{1}=\frac{\exp \left(-\varepsilon / T_{H}\right)}{1+\exp \left(-\varepsilon / T_{H}\right)} ; k_{b}=1$. As the two level system (TLS) is virtually a part of the heat bath, we refer to the combined object as a heat source hereafter. It is important to note that the TLS is held in isothermal conditions: it is tightly connected to a large thermal reservoir at $T_{H}$. Thus, the engine absorbs energy from the TLS, yet the TLS does not cool down to zero, as its temperature is kept fixed. This is analogous to the process of reversible isothermal expansion of ideal gases. We emphasize that our results do not depend on the particular choice of the subsystem, and the TLS model is introduced here as a simple demonstration.

The right half of the plot shows an automatic machine, referred to as the engine. It consists four components: detector, communication channel, processor and receiver. Following Shanon general model for a communication system [8], in a working cycle the detector detects the state of the heat source, encodes it, and sends this information through the transmission channel to the processor which will decode it. Based on this information, the processor sends an instruction to properly set the receiver, to accept the energy of the subsystem. While a measurement of the heat source state and receiving its energy can be done (virtually) without energy consumption 2], energy dissipation in the engine is attributed to the transmission of information in the channels and the resulting setup of the receiver, corresponding to the Landauer erasure principle [2].

Analysis of performance. - In each working cycle the engine needs to acquire the information on the state of the finite subsystem. The total amount of information that has to be absorbed during this process is given by

$$
I=\frac{\bar{E}}{T_{H}},
$$

where, e.g., $\bar{E}=P_{0} \varepsilon_{0}+P_{1} \varepsilon_{1}$ is the average energy of the TLS subsystem. This information has to be transferred through the engine communication channel. The measure for a communication system is the channel capacity $I_{p}\left(S, N_{0}\right)$, describing the maximum number of bits that a system can communicate reliably per channel use. Here $S$ and $N_{0}$ (a function of $T_{C}$ ) are the pulse and noise average energies, respectively [9]. In order to transfer $I$ on the status of the heat source to the engine we need to invest energy

$$
Q \equiv \frac{I}{I_{p}} S,
$$

which cannot be refunded (else it contradicts the maximum capacity theorem), thus this is the heat dissipated in the engine during an operation cycle. The engine efficiency is given by the amount of available working energy over the invested energy. Using $W=\bar{E}-Q$ for the work attained per cycle, we obtain

$$
\eta=\frac{W}{\bar{E}}=\frac{\bar{E}-Q}{\bar{E}}=1-\frac{S}{T_{H} I_{p}} .
$$

We will optimize the engine cycle (i) with respect to its efficiency, minimizing $Q$, and (ii) with respect to its power $P=W / n$, where $W=\bar{E}-Q$ is the work attained per cycle. Here $n=I / I_{p}$, the number of pulses that are needed to transfer the information $I$ in a working cycle, is proportional to the period of the engine. Utilizing Eqs. (31) and (41) we get

$$
P=T_{H} I_{p}-S
$$

Maximizing the power with respect to the pulse power $S$ and substituting the optimal value $S_{M}$ into (5), provides the maximum power efficiency

$$
\eta_{M}=1-S_{M} / T_{H} I_{p}\left(S_{M}, T_{C}\right) .
$$

We consider next classical and quantum channels, derive $\eta$ for the two scenarios mentioned above, and manifest that they independently follow a universal behavior.

Case I: Gaussian Channel. - Suppose we send information over a classical continuous memoryless channel of bandwidth $B$ (measured in Hertz), subjected to an additive white Gaussian noise with a power spectral density $N_{0}$. Assuming a signal power $\mathcal{S}$, and taking $B N_{0}$ as the total noise power in a bandwidth $B$, one obtains the Shanon-Hartley capacity $C=B \ln \left(1+\frac{\mathcal{S}}{B N_{0}}\right)$ [8]. Defining $S=\mathcal{S} / B$ as the average energy carried by the pulse and $I_{p}=C / B$ as a dimensionless capacity [9], we get

$$
I_{p}=\ln \left(1+\frac{S}{N_{0}}\right) .
$$

In order to transfer the information $I$ on the status of the subsystem into the engine we invest $Q=I \frac{S}{\ln \left(1+\frac{S}{T_{C}}\right)}$ [see (4)], identifying $N_{0}=T_{C}$ as the thermal noise power spectral density.

Carnot efficiency. - The minimum value of the last relation is $Q=I T_{C}$, obtained when $S \rightarrow 0$, i.e., when the engine works reversibly with minimal energy consumption. As we invest $Q$ and gain $\bar{E}$, the net average energy gain of the engine is

$$
W=\bar{E}-I T_{C}=\bar{E}\left(1-T_{C} / T_{H}\right),
$$

resulting in the maximal efficiency $\eta_{C}=1-T_{C} / T_{H}$ [10].

Maximum power efficiency. - We calculate $\eta_{M}$ using the Gaussian capacity (8). The power (6) reduces to

$$
P=T_{H} \ln \left(1+S / T_{C}\right)-S .
$$

Taking $\frac{\partial P}{\partial S}=0$ yields the energy per pulse maximizing the power, $S_{M}=T_{H}-T_{C}$. We substitute this result 


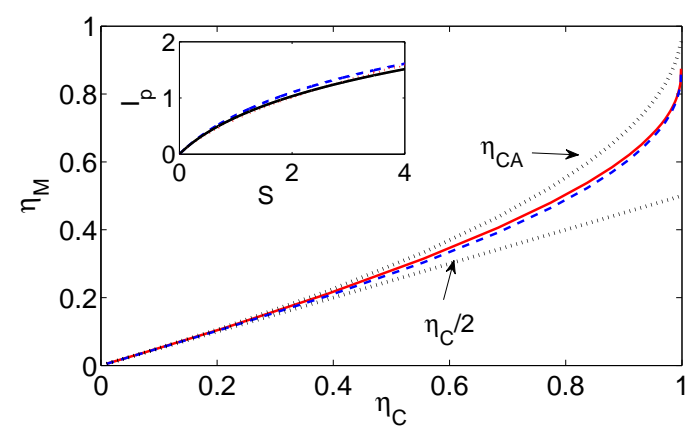

FIG. 2: Maximum power efficiency for a heat engine of a narrowband bosonic channel with $\nu=0.2$ (full) and $\nu=2$ (dashed). $T_{C}=1$ was kept fixed. The CA efficiency and half of the Carnot limit are shown as dotted lines. Inset: Gaussian (dashed line), wideband bosonic (dotted line) and narrowband bosonic with $\nu=2$ (full line) capacities, $T_{C}=1$.

into (77) and obtain $\eta_{M}=1-S_{M} /\left[T_{H} \ln \left(1+\frac{S_{M}}{T_{C}}\right)\right]$. In terms of the Carnot efficiency, $\eta_{C}=\frac{S_{M}}{T_{H}}$, we find that

$$
\begin{aligned}
\frac{\eta_{M}}{\eta_{C}} & =\frac{1}{\eta_{C}}+\frac{1}{\ln \left(1-\eta_{C}\right)} \\
& =\frac{1}{2}+\frac{1}{12} \eta_{C}+\frac{1}{24} \eta_{C}^{2}+\mathcal{O}\left(\eta_{C}^{3}\right) .
\end{aligned}
$$

For $\eta_{C} \rightarrow 1$, the maximal power efficiency converges to 1 . Note that the coefficient of the second (quadratic) term is smaller than the value recovered in several spatiallysymmetric systems [11].

Case II: Bosonic Channel. - We next send the same classical information, yet in the form of quantum states, over a memoryless quantum channel which is a bosonic field (e.g. an electromagnetic radiation): The message information is encoded into modes of frequency $\nu$ and average photon number $n_{B}(\nu)$ [12, 13]. The signal power is denoted by $\mathcal{S}$; noise power is $N=\pi T_{C}^{2} / 12 \hbar$ 14]. Under the constraint that the message-ensembleaveraged energy of the channel is fixed, the capacity of this channel is given by $C=\frac{\pi}{6 \hbar}\left(T_{e}-T_{C}\right)$, where $T_{e}$, the (effective) temperature of the decoder, is defined through the relation $\mathcal{S}+\frac{\pi T_{C}^{2}}{12 \hbar}=\frac{\pi T_{e}^{2}}{12 \hbar}$, leading to $C=$ $\frac{\pi T_{C}}{6 \hbar}\left[\left(12 \hbar \mathcal{S} / \pi T_{C}^{2}+1\right)^{1 / 2}-1\right]$. Redefining $I_{p}=C / B$ and $S=\mathcal{S} / B ; B$ denotes an effective bandwidth, we obtain

$$
I_{p}=\frac{\pi T_{C}}{6 B \hbar}\left[\left(\frac{12 \hbar S B}{\pi T_{C}^{2}}+1\right)^{1 / 2}-1\right] .
$$

Remarkably, this capacity is inherently linked to the thermal conductance $\kappa$ of a ballistic quantum wire [14].

Carnot efficiency. - We again minimize heat production in the channel $Q=I S / I_{p}$, using (3) and (12). The minimum value of this expression is $(\lambda \equiv 6 B \hbar / \pi)$

$$
\begin{aligned}
Q_{S \rightarrow 0} & =\lim _{S \rightarrow 0} \frac{\bar{E}}{T_{H}} \frac{\lambda S / T_{C}}{\left(2 \lambda S / T_{C}^{2}+1\right)^{1 / 2}-1} \\
& =\bar{E} T_{C} / T_{H},
\end{aligned}
$$

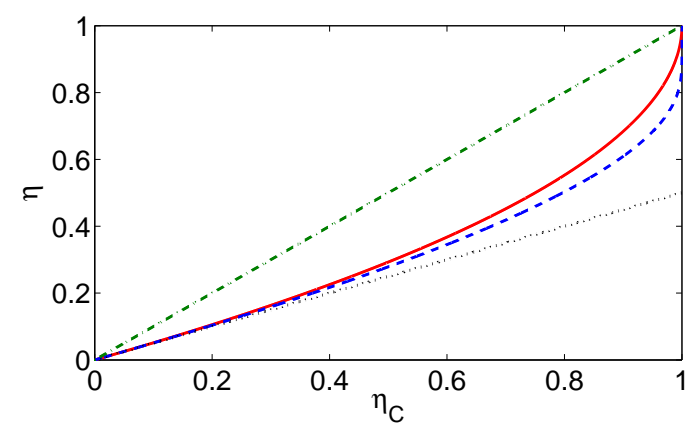

FIG. 3: Maximum power efficiency using different models for the information channel: Gaussian (8) (dashed line), wideband Bosonic (12) (dotted), compared to the Curzon-Ahlborn efficiency (full) and the Carnot efficiency (dashed-dotted line).

retrieving the Carnot limit, $\eta_{C}=\left(\bar{E}-Q_{S \rightarrow 0}\right) / \bar{E}=(1-$ $\left.T_{C} / T_{H}\right)$.

Maximum power efficiency. - Maximizing $P$, we obtain $S_{M}=\frac{T_{C}^{2}}{2 \lambda}\left[\left(\frac{T_{H}}{T_{C}}\right)^{2}-1\right]$. We plug it into (7) and exactly resolve only the linear term

$$
\eta_{M} / \eta_{C}=1 / 2
$$

Interestingly, this result holds in both the high temperature limit, when the classical Shanon capacity is recovered $I_{p}=S / T_{C}$, as well as at low temperatures when quantum effects become important and the channel capacity approaches $I_{p}=\sqrt{\pi S / 3 \hbar B}$.

We proceed and explore a narrowband photon channel, $B \ll \nu$, where $\nu$ is the channel central frequency. In this case the noise power is given by $N=h \nu n_{B}\left(\nu, T_{C}\right) B$; $n_{B}(\nu, T)=\left[e^{h \nu / T}-1\right]^{-1}$ is the Bose-Einstein distribution. Based on the assumption that photon noise is additive, the (dimensionless) capacity $I_{p}=C / B$ fulfills [8]

$$
\begin{aligned}
& I_{p}=\ln \left[1+\frac{S}{h \nu}\left(1-e^{-h \nu / T_{C}}\right)\right]+\left[S / h \nu+n_{B}\left(\nu, T_{C}\right)\right] \\
& \times \ln \left[1+\frac{h \nu}{S+h \nu n_{B}\left(\nu, T_{C}\right)}\right]-n_{B}\left(\nu, T_{C}\right) h \nu / T_{C} .(15)
\end{aligned}
$$

Since analytical results are cumbersome, we acquire $S_{M}$ numerically, seeking the intersect of $1 / T_{H}$ with $\partial I_{p}(S) / \partial S$, see Eq. (6). The results, displayed in Fig. 2. clearly demonstrate that $\eta_{M}$ is bounded by $\eta_{C A}$ from above, and that $\eta_{M}=\eta_{C} / 2$ in the lowest order of $\Delta T / T$. Interestingly, the results are almost independent of the channel central frequency $\nu$. The inset displays channel capacities for the three models considered: Gaussian, wideband and narrowband bosonic channels. Figure 3 further shows the maximum power efficiency $\eta_{M}$ for the classical and quantum wideband channels, as a function of $\eta_{C}$, compared to the CA and the Carnot efficiencies. We find that the CA value is an upper bound for $\eta_{M}$.

Case III: Generic Channel. - Motivated by the classical-Gaussian channel and the quantum models, we 
consider next a general form for a channel capacity,

$$
I_{p}=\alpha x+\beta x^{2}+\gamma x^{3}+\mathcal{O}\left(x^{4}\right) .
$$

Here $x=S / T_{C}$ is the signal power over noise, and the parameters $\alpha, \beta$ and $\gamma$ may depend on $T_{C}$. These coefficients are constricted so as to produce positive capacity, $I_{p} \geq 0$. Assuming $I_{p}$ is a concave function, valid for the cases investigated above, the inequality $3 \gamma S<-\beta T_{C}$ must be satisfied. Since $S>0$ and $\gamma>0$ [so as $I_{p}(S \gg 0)>0$, we conclude that $\beta$ must be negative. In particular, for the Gaussian channel (8) a power expansion gives $\alpha=1, \beta=-1$ and $\gamma=4 / 3$, while for the bosonic wideband quantum channel we get $\alpha=1$, $\beta=-3 \hbar / \pi T_{C}$ and $\gamma=18 \hbar^{2} / \pi^{2} T_{C}^{2}$. Using the generic form (16), the maximal efficiency is obtained by minimizing heat production, $Q=I S / I_{p}$, resulting in

$$
Q(S \rightarrow 0)=I T_{C} / \alpha ; \quad \eta=\tilde{\eta}_{C} / \alpha,
$$

where $\tilde{\eta}_{C}=\eta_{C}+\alpha-1$. Thus, in order to reach the Carnot limit one must require that $\alpha=1$. Next, still using the form (16) we maximize $P$ and obtain the pulse power $S_{M}=\frac{-\beta T_{C}}{3 \gamma}\left[1-\left(1-\frac{3 \gamma \tilde{\eta}_{C}}{\beta^{2}}\right)^{1 / 2}\right]$. Plugging this value into (7) leads to

$$
\eta_{M}=\frac{\tilde{\eta}_{C}}{2 \alpha}+\frac{\tilde{\eta}_{C}^{2}}{4}\left(\frac{1}{\alpha^{2}}-\frac{\gamma}{2 \beta^{2} \alpha}\right)+\mathcal{O}\left(\tilde{\eta}_{C}^{3}\right) .
$$

Imposing $\alpha=1$ results in

$$
\eta_{M} / \eta_{C}=\frac{1}{2}+\frac{\eta_{C}}{4}\left(1-\gamma / 2 \beta^{2}\right)+\mathcal{O}\left(\eta_{C}^{2}\right) .
$$

The following important observations can be made: (i) The Carnot limit $\eta_{C}=\left(T_{H}-T_{C}\right) / T_{H}$ and the efficiency at maximum power $\eta_{M}=\eta_{C} / 2$ (at first order in $\eta_{C}$ ) are non-subordinate elemental results in thermodynamics: Demanding that $\alpha=1$ for channels working at maximal capacity, independently produces both the Carnot efficiency, and the universal linear coefficient in Eq. (19). (ii) A universal relation between the two efficiencies stands still even below the upper bound: Assuming that $\alpha<1$ accounts for loss mechanisms in the channel, operating below its full capacity, the maximal efficiency of the engine is given by $\tilde{\eta} / \alpha$, while the maximal power efficiency (in linear response) is exactly half this value, $\tilde{\eta} / 2 \alpha$. (iii) The first (linear) term in (18) is independent of the details of the channel model, $\beta$ and $\gamma$. (iv) Finally, the (lack of) universality in the quadratic term of $\eta_{M}$ has been a source of debates [11]. Our analysis exposes that this coefficient depends on the details of the channel, i.e., on the relations between the nonlinear terms. In particular, if $\gamma=2 \beta^{2}$ the quadratic term diminishes, in agreement with the quantum (wideband) bosonic channel. When $4 \beta^{2}=3 \gamma$, it reduces to $\eta_{C}^{2} / 12$, as in the classical Gaussian channel. For $\gamma=\beta^{2}$ the quadratic term becomes $\eta_{C}^{2} / 8$, recovering the CA result (2) and other symmetric models [11.

Summary. - We have designed here a minimal model of a heat engine (or a Maxwell's demon), attributing energy dissipation within the machine to irreversible loss of energy within the engine communication channels. Analyzing both classical and quantum information channels we have demonstrated that our model satisfies the Carnot limit for reversible operation mode. Independently, it leads to the universal linear term in the maximum power efficiency, exhibiting the existence of fundamental laws for finite-time thermodynamic processes. While the universality of the maximal power efficiency in the linear response regime has been explained based on the Onsager's symmetry [5], our model establishes this universality developing a distinct, information based picture. We expect this prototype model to be immensely useful for studying other basic concepts in thermodynamics. For example, the engine described here could serve as a cooling device, or the performance could be analyzed for a subsystem away from the Boltzmann distribution. One could also advance the model beyond the strong coupling limit, $W \propto \bar{E}$ [15], or consider other sources of dissipation. Finally, it is of great interest to further include quantum effects, considering transmission of quantum information.

Acknowledgments. This research has been supported by NSERC.
[1] R. Landauer, IBM J. Res. Dev. 5, 183 (1961).

[2] K. Maruyama, F. Nori, and V. Vedral, Rev. Mod. Phys. 81, 1 (2009), and references therein.

[3] R. S. Berry, V. A. Kazakov, S. Sieniutycz, Z. Szwast, and A. M. Tsirlin, Thermodynamic Optimization of FiniteTime Processes (Wiley, Chichester, U.K., 2000).

[4] F. Curzon and B. Ahlborn, Am. J. Phys. 43, 22 (1975).

[5] C. Van den Broeck, Phys. Rev. Lett. 95, 190602 (2005).

[6] A. E. Allahverdyan, R. S. Johal, and G. Mahler, Phys. Rev. E 77, 041118 (2008).

[7] T. Schmiedl and U. Seifert, Europhys. Lett. 81, 20003
(2008)

[8] F. T. S. Yu, Entropy and Information Optics (MarcelDekker, New-York), 2000.

[9] Standardly, the units of the channel capacity $C$ are nats/sec, and it is given in terms of the signal power $\mathcal{S}$ and the noise power $N$. Here $I_{p}$ is a dimensionless channel capacity, given in terms of the signal and noise averaged energy, $S$ and $N_{0}$, respectively. For simplicity, we use 2 pulses as the basic unit for counting information transfer in a channel [8]. This choice does not affect our results.

[10] The model could be also formulated using a one-molecule 
gas. In an engine cycle it is reversibly compressed to the volume $V / 2$ at temperature $T_{C}$, then expanded back to $V$ at $T_{H}$. In the compression phase the information $I=$ $\ln (2)$ is obtained with an energy cost $Q=T_{C} \ln (2)$. The information is used in the expansion phase to gain $\bar{E}=$ $T_{H} \ln (2)$, yielding the Carnot efficiency.

[11] M. Esposito, K. Lindenberg, and C. Van den Broeck, Phys. Rev. Lett. 102 , 130602 (2009).
[12] C. M. Caves and P. D. Drummond, Rev. Mod. Phys. 66, 481 (1994).

[13] V. Vedral, Rev. Mod. Phys. 74, 197 (2002).

[14] M. Blencowe, V. Vitelli, Phys. Rev. A 62, 052104 (2000).

[15] We refer to this engine as "strongly coupled" since the work attained $\bar{E}-Q \propto I$ is proportional to the energy pumped, $\bar{E}=I T_{H}$ (3). 